Sharif University of Technology
Scientia Iranica
Transactions E: Industrial Engineering
http://scientiairanica.sharif.edu
IRA I ENTIA

\title{
A new nondominated sorting genetic algorithm based on the regression line for fuzzy traffic signal optimization problem
}

\author{
H. Asadi ${ }^{a}$, R. Tavakkoli Moghaddam ${ }^{\mathrm{b}}$, N. Shahsavari Pour ${ }^{\mathrm{c}}$, and E. Najafia,* \\ a. Department of Industrial Engineering, Science and Research Branch, Islamic Azad University, Tehran, Iran. \\ b. School of Industrial Engineering, College of Engineering, University of Tehran, Tehran, Iran. \\ c. Department of Industrial Technology and Management, Vali-e-Asr University of Rafsanjan Iran.
}

Received 2 December 2015; received in revised form 20 December 2016; accepted 7 January 2017

\author{
KEYWORDS \\ Traffic signal systems; \\ Genetic algorithm; \\ Vehicle and pedestrian \\ delays; \\ ANOVA.
}

\begin{abstract}
Traffic jam is a daily problem in nearly all major cities in the world and continues to increase with population and economic growth of urban areas. Traffic lights, as one of the key components at intersections, play an important role in control of traffic flow. Hence, study and research on phase synchronization and time optimization of the traffic lights could be an important step to avoid creating congestion and rejection queues in a urban network. Here, we describe the application of NSGA-II, a multi-objective evolutionary algorithm, to optimize both vehicle and pedestrian delays in an individual intersection. In this paper, we improve NSGA-II algorithm based on the regression line to find a Pareto-optimal solution or a restrictive set of Pareto-optimal solutions based on our solution approaches to the problem, named PDNSGA (Non-dominated Sorting Genetic Algorithm based on Perpendicular Distance). The high speed of the proposed algorithm and its quick convergence makes it desirable for large scheduling with a large number of phases. It is demonstrated that our proposed algorithm (PDNSGA) gives better outputs than those of MOGA, NSGA-II, and WBGA in traffic signal optimization problem, statistically .

(C) 2018 Sharif University of Technology. All rights reserved.
\end{abstract}

\section{Introduction}

Traffic congestion is a daily problem in nearly all major cities in the world and continues to increase with population and economic growth of urban areas. The increasing traffic demand strains the existing transportation system, especially when the network is oversaturated during peak hours. Oversaturation occurs when the queues of vehicles on a receiving street interfere with the performance of the respective adjacent upstream streets, and though these conditions

*. Corresponding author.

E-mail address: najafi1515@yahoo.com (E. Najafi) may last for only a short time, the time to clear the network may be significant. Costs of infrastructure renewal and expansion may be cost-prohibitive, and under limited budgets, viable strategies are required to enhance the mobility and efficiency of the existing traffic without investing in new infrastructure.

There are two objectives for this research. We develop an effective procedure to optimize signal timing of an individual intersection by minimizing both vehicle and pedestrian delays. Traffic signals aim generally to minimize average vehicle delay, but pedestrian delay is not taken into account. Such a strategy is reasonable for rural areas or highways where very few pedestrians interfere with vehicular traffic. However, in a central business district with a lot of pedestrians walking around, the strategy that only optimizes vehicle flows 
would not be suitable, because the pedestrian delay is ignored. Ignoring pedestrian delay might even result in people choosing to use vehicles more frequently than walking. Therefore, when pedestrian flows start to have influence on vehicle flows, pedestrian signal plans should be optimized considering delays for both groups. Traffic signal plan optimization should be a trade-off between vehicle and pedestrian delays by minimizing travel delay for all the travelers.

Ishaque and Noland [1] analyzed the effect of signal cycle timing on both vehicle and pedestrian delays in a hypothesized network by a VISSIM microsimulation model. Aiming to minimize the multimodal travel delay and travel costs, they found that optimal cycle lengths under light traffic conditions (60 to 72 seconds) were shorter than optimal cycle lengths under heavy traffic conditions (90 seconds). However, they only discussed eight fixed-time non-coordinated signal plans with single or double exclusive pedestrian phases. In addition, pedestrian compliance effect was not considered in the research. Based on their research in 2005, Ishaque and Noland [2] studied trade-offs between pedestrian and vehicle traffics in the same hypothetical network by a VISSIM microsimulation model. Aiming to optimize average travel cost per person in all modes of the network, they found that shorter cycle lengths were beneficial for pedestrians, and that signal plans advantageous to vehicles might be disadvantageous to pedestrians. Based on different proportions of pedestrians to vehicle users and different pedestrian time values, suitability of three different pedestrian phase types was analyzed so that the optimal network performance could be achieved for all road users. Compared with their previous research, pedestrian compliance effect was considered in the research, and a variety of signal plans were improved. However, the variety was still limited to two-phase vehicle signal plans with single exclusive or double exclusive, or staggered pedestrian crossing phase(s). A deterministic model [3,4] was proposed that incorporated both pedestrian and vehicle delays into a signal coordination plan. The author(s) analyzed the running results of the model on a hypothesized five-intersection arterial with various offsets, and found that the best offsets for vehicles and pedestrians along the arterial were not necessarily the same. In order to minimize total pedestrian and vehicle user cost, an optimal signal coordination plan could be achieved by balancing between pedestrian and vehicular delays. Li et al. [5] developed a traffic signal optimization strategy, programmed in Matlab, for an individual intersection to minimize weighted total vehicle and pedestrian delays. The total vehicle and pedestrian delays on sidewalk were calculated based on their deterministic queuing model, respectively. Total pedestrian delay on crosswalk was calculated based on an empirical pedestrian speed model, which considered interactions between pedestrian platoons. Mehan [6] and Barzegar [7] worked on traffic signal control for an isolated intersection signal (adjacent intersection) with fuzzy controller methods.

Gokulan and Srinivasan [8] adopted a type-2 fuzzy set and designed a distributed multi-agent trafficresponsive signal control system. This system was tested on virtual road networks with several scenarios. Results showed the superior performance of the approach in handling unplanned and planned incidents and obstructions.

Recently, metaheuristic algorithms [9] have become very popular as optimization methods for solving traffic light scheduling problems. A first attempt corresponds to the study of Rouphail et al. where a Genetic Algorithm (GA) was coupled with the CORSIM [10] microsimulator for the timing optimization of nine intersections in the city of Chicago (USA). The results, in terms of total queue size, were limited due to the delayed convergence behavior of the GA. Turky et al. [11] used a GA to improve the performance of traffic lights and pedestrians crossing control in a unique intersection with a four-way two-lane junction. The algorithm solved the limitations of traditional fixedtime control for passing vehicles and pedestrians, and it employed a dynamic control system to monitor two sets of parameters.

Odeh et al. [12] presented a hybrid algorithm that combines Fuzzy Logic Controller (FLC) and Genetic Algorithms (GAs) and its application on a traffic signal system. It has been used to adopt the decision rules of FLCs that define an intelligent traffic signal system, obtaining a higher performance compared to that of a classical FLC-based control. The simulation results yielded by the hybrid algorithm show an improvement of up to $34 \%$ in the performance with respect to a standard traffic signal controller. Another signal control methodology is formulated as a quadratic programming problem to minimize and balance the link queues, thus minimizing the risk of queue spillback [13]. Göttlich et al. [14] presented a numerical approach to the optimization of switching points as a function of time based upon the macroscopic traffic flow model. The numerical discussion relies on an equivalent reformulation of the original problem as well as a mixed-integer discretization of the flow dynamics. The large-scale optimization problem is solved using derived heuristics within the optimization process. Collotta et al. [15] proposed a novel approach to managing dynamically the traffic lights cycles and phases in an isolated intersection. The target of the work is a system that, compared with previous solutions, offers improved performance, which is flexible and can be implemented on off-the-shelf components. The proposed system combines the advantages of the 
WSN, such as easy deployment and maintenance, flexibility, low cost, noninvasiveness, and scalability, with the benefits of using four parallel fuzzy controllers, i.e. better performance, fault-tolerance, and support for phase-specific management.

Shahsavari Pour et al. [16] presented a new fuzzy multi-objective mathematical model to minimize the length of traffic queue and pedestrian delays to optimize the control of fluctuating traffic volumes such as oversaturated or unusual load conditions. The problem is solved by genetic algorithm, and a new defuzzification method is introduced. Duerr [17] used a GA with a microscopic traffic simulator as the fitness evaluator to minimize the Performance Index (PI) which considered vehicle behavior at intersections and transit stops. The optimization results of a seven-node arterial in Würzburg (Germany) and temporal deviation of each phase duration from the standard setting at each node showed that travel time dropped $25 \%$ and $5 \%$ for buses and cars, respectively. Furthermore, so as to optimize signal control under oversaturated traffic condition, Girianna and Benekohal $[18,19]$ applied a GA to a grid network of arterials. The optimization results of a hypothesized twenty-node network and green time of each phase at an intersection showed that queues were successfully distributed spatially over different intersections and temporarily over different signal cycles.

In this paper, we apply a new multi-objective genetic algorithm to find a Pareto-optimal solution or a restrictive set of Pareto-optimal solutions based on our solution approaches to the problem. In this paper, our purpose is to present a solution methodology to obtain all Pareto-optimal solutions to optimize signal timing and enable the decision-makers to evaluate a greater number of alternative solutions. The purpose of this study is to extend this methodology to reach a solution to multi-objective optimal control problems under the framework of NSGA-II.

This paper is organized as follows. In Section 2, we present the problem definition and formulation. In Section 3, a solution procedure is introduced. We develop an algorithm, namely PDNSGA. To illustrate the proposed approach, a number of examples are presented in Section 4. The related results of this algorithm are analyzed by the analysis of variance (ANOVA) method in Section 5. Finally, conclusion is given in Section 6 .

\section{Multi-objective mathematical model}

Traffic signal timing planning is a typical multiobjective optimization problem; for a signalized system, an optimal timing plan is usually required to meet four typical objectives (minimizing delay, minimizing stops, minimizing fuel consumption, and maximizing progression). The problem discussed in this paper is minimizing the queue length on each side of an intersection during peak hours with fuzzy entrance and exit rates on each side of the intersection. Generally, traffic signals aim to minimize average vehicle delay, but pedestrian delay is not taken into account. Such a strategy is reasonable for rural areas or highways where very few pedestrians interfere with vehicular traffic. However, in a central business district with a lot of pedestrians walking around, the strategy that only optimizes vehicle flows would not be suitable because the pedestrian delay is ignored. Ignoring pedestrian delay might even result in people choosing to use vehicles more frequently than walking. Traffic signal plan optimization should be a trade-off between vehicle and pedestrian delays by minimizing travel delay for all the travelers. Therefore, when pedestrian flows start to have an influence on vehicle flows, pedestrian signal plans should be optimized considering delays for both groups.

Fuzzy logic is a powerful tool for processing nondeterministic and non-linear problems. It can represent fuzzy and qualitative knowledge; therefore, it can imitate human's reason. As we know, a seasoned traffic police can handle traffic quickly and effectively.

Actually, the reasons and decisions are made merely by the use of interrelated qualitative knowledge. The process of reasoning and decision-making can be described as follows: For a lane, if there are many vehicles arriving in, more green time is allocated. Otherwise, less green time is allocated or the phase turns to the next one. Of cause, it is necessary to simultaneously consider traffic demands in other lanes when changing the phase. The control process is shown in Figure 1. The traffic intensity can be denoted by

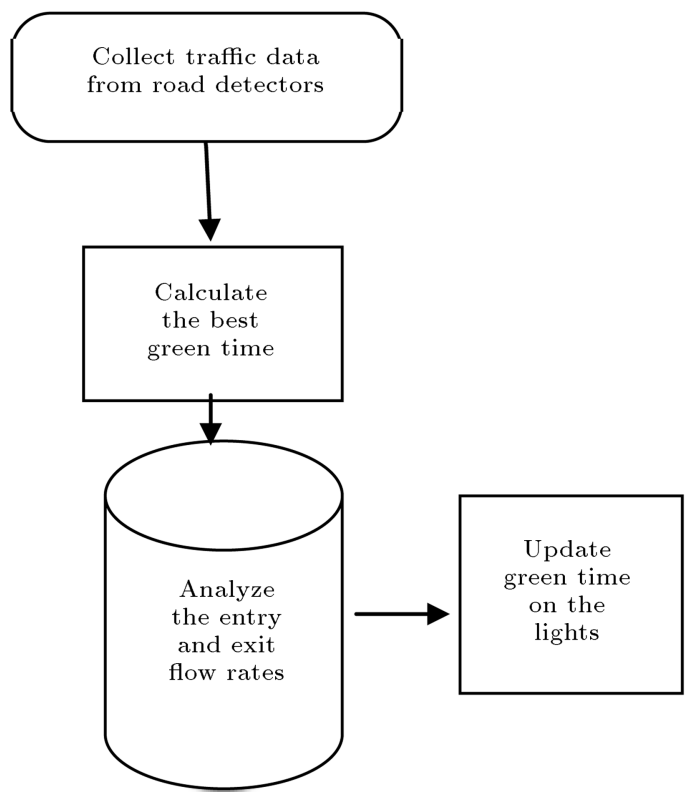

Figure 1. Scheme of traffic optimization process using data collected from road detectors. 
queue length before stop line or traffic density on the approaches in the current or next phase.

Traffic signal timing changes at different hours of day, and the amount of traffic on each street and the intersection is used to help increase the efficiency of lights. Although this method has improved the controlling process of intersections, in a very busy intersection and in cities that have particular traffic treatment, the control of intersection will face this problems. So, we need to have a system that responds to variations of the traffic patterns quickly. This study is made to optimize green time duration in order to minimize traffic jam length. Prior to the optimization process, road-detector should gather data related to intersection traffic. These data are required to make decisions if current conditions are sufficient, or they have to be improved (see Figure 1).

For the sake of clarification, we have conducted a sensitivity analysis on the traffic light at a two-phase intersection, and then it has been developed for a fourphase traffic problem. When phase I has red time (T1), traffic jam length in this phase has an increasing rate. On the contrary, traffic jam length of phase II with green time has a decreasing rate. Red time (WALK time) in each phase is the sum of green time in other phases which increases as the green time devoted to each phase increases; therefore, jam length in other phases may also increase.

The pedestrian's queue length has an incremental rate and reaches its highest level at the end of green time (points A1, and A2). As soon as intersection's red time starts, all the pedestrians cross the intersection immediately (WALK time). As a result, pedestrians' queue is equal to zero (see Figure 2). In addition, the model considers the compliance effect of pedestrians. In order to avoid waiting for the next WALK interval, certain pedestrians increase their waking speed and begin their crossing without a WALK indication. The majority of these phenomena happen during the flashing DONT WALK time. The aim of this research is to obtain the optimal traffic light green and red time to minimize the fuzzy queue length of vehicles

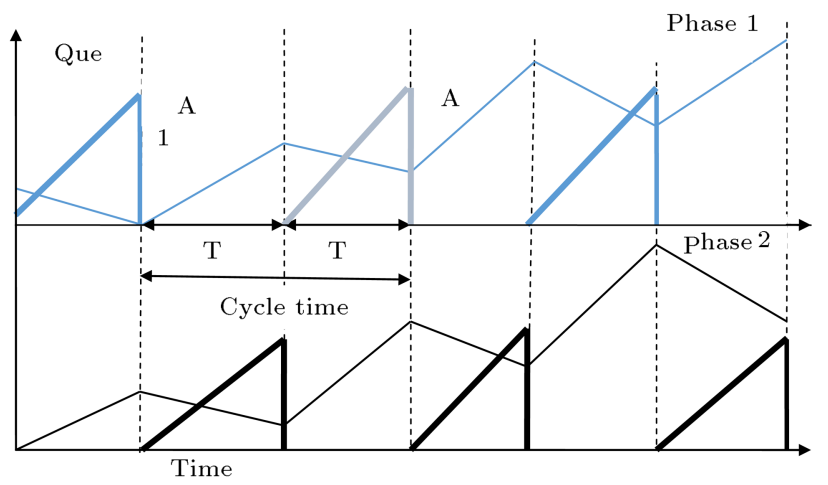

Figure 2. Sensitivity analysis for two-phase intersection. and pedestrians. The notations used for the problem formulation are as follows:

$L_{i} \quad$ The lower bound of the green time in

The number of phases in an intersection. phase $i$.

$U_{i} \quad$ The upper bound of the green time in phase $i$.

$\widetilde{T I R}_{i} \quad$ The fuzzy entrance flow rate of cars in red time duration in phase $i$.

$\widetilde{T I G}_{i} \quad$ The fuzzy entrance flow rate of cars in green time duration in phase $i$.

$T \tilde{O} G_{i} \quad$ The fuzzy exit flow rate in green time duration in phase $i$.

$N \widetilde{T I} R_{i} \quad$ The fuzzy entrance cars in red time duration in phase $i$.

$N \widetilde{T I} G_{i} \quad$ The fuzzy entrance cars in green time duration in phase $i$.

$\widehat{N T O} G_{i} \quad$ The fuzzy exit cars in green time duration in phase $i$.

$\widetilde{T I P}_{i} \quad$ The fuzzy entrance rate of pedestrians in red time duration (DONT WALK) in phase $i$.

$N \widetilde{T I} P_{i} \quad$ The fuzzy entrance pedestrians in red time duration in phase $i$.

$T S \quad$ Duration of the analysis period.

$T R_{i} \quad$ The red time duration in phase $i$.

$T G_{i} \quad$ The green time duration in phase $i$.

$C T_{i} \quad$ The cycle time duration in phase $i$.

$N C T_{i} \quad$ Number of cycles time in the TS.

$\widetilde{Q R}_{(i, j)} \quad$ The fuzzy queue length when red time duration in phase $i$ and the $j$ th cycle time is ended.

$\tilde{Q} G_{(i, j)} \quad$ The fuzzy queue length when green time duration in phase $i$ and the $j$ th cycle time is ended.

The problem is formulated as in the following model presented by Asadi and Shahsavari [16]:

$$
\begin{aligned}
\operatorname{Min} Q 1= & \sum_{i=1}^{n}\left(N \tilde{T} I R_{i}+\left(N \tilde{C} T_{i}-1\right)\right. \\
& \left.\times\left(N \tilde{T} I R_{i}+N \tilde{T} I G_{i}-\widehat{N T O G_{i}}\right)\right), \\
\operatorname{Min} Q 2= & \sum_{i=1}^{n}\left(T \tilde{I} P_{i} \times T R_{i}\right) .
\end{aligned}
$$

Subject to: 


$$
\begin{aligned}
& L_{i} \leq T G_{i} \leq U_{i}, \\
& T R_{i}-\left(\sum_{i=1}^{n} T G_{i}\right)-T G_{i} \quad \forall i, i=1,2, \ldots, n, \\
& N \tilde{T I} R_{i}-T \tilde{I} R_{i} \times T R_{i} \quad \forall i, i=1,2, \ldots, n, \\
& N \widetilde{T I} G_{i}=\widetilde{T I} G_{i} \times T G_{i} \quad \forall i, i=1,2, \ldots, n, \\
& N \widetilde{T O G_{i}}=\widetilde{T O} G_{i} \times T G_{i} \quad \forall i, i=1,2, \ldots, n, \\
& C T_{i}=T R_{i}+T G_{i} \quad \forall i, i=1,2, \ldots, n, \\
& N C T_{i}=T S / C T_{i} \quad \forall i, i=1,2, \ldots, n, \\
& \widetilde{Q R} \widetilde{Q T}_{(i, j)}+N \widetilde{T I} R_{i} \quad \forall i, i=1,2, \ldots, n .
\end{aligned}
$$

Eq. (1) minimizes the total queue length of vehicles in all phases of the intersection; Eq. (2) minimizes the total queue length of pedestrians in all phases of the intersection. Eq. (3) restricts green time per phase to take a value within a lower bound $L_{i}$ and an upper bound $U_{i}$. Eq. (4) represents the red time duration in phase $i$; Eq. (5) represents the fuzzy entrance cars in red time duration in phase $i$.

Eq. (6) represents the fuzzy entry cars in green time duration in phase $i$. Eq. (7) represents the fuzzy exit cars in green time duration in phase $i$. Eq. (8) shows that the cycle time duration in per phases is equal to the total green and red time. Eq. (9) represents the number of cycle time per phases. When green time duration in phase $i$ and $j$ th cycle time is ended, the fuzzy queue length is calculated by Eq. (9). If the last cycle time duration is equal to $m$ in the Time period Studied (TS), the objective function in Eq. (1) will minimize $\widetilde{Q R}_{(i, m)}$.

There are various solution approaches to solve the multi-objective problem. Among the most widely used techniques are sequential optimization, e-constraint method, weighting method, goal programming, goal attainment, and distance-based and direction-based methods. In this study, we also proposed a novel genetic algorithm to obtain Pareto-optimal solutions based on the distance to the regression line (GADRL) for this problem.

\section{Novel genetic algorithm}

Evolutionary algorithms for Multi-Objective (MO) optimization are currently gaining significant attentions from researchers in various fields due to their effectiveness and robustness in searching for a set of global trade-off solutions. This growing interest is reflected by the significantly increasing number of different evolutionary-based approaches and variations of the existing method published in technical literatures. The purpose of this study is to extend this methodology to obtain the solution to multi-objective optimal control problems under the framework of NSGA-II. The Non-Dominated Sorting Genetic Algorithm (NSGA) proposed by Srinivas and Deb [20] was one of the first evolutionary algorithms for solving multi-objective optimization problems. Although NSGA has been successfully applied to solving many problems, the main criticisms of this approach have been its high calculation's volume of nondominated sorting, lack of elitism, and the need for specifying a tunable parameter called sharing parameter. Recently, Deb et al. [21] reported an improved version of NSGA, called NSGAII, to address all the above issues.

\subsection{Multi-objective optimization and genetic algorithms}

Being a population-based approach, GA is well suited to solve multi-objective optimization problems. A generic single-objective GA can be modified to find a set of multiple non-dominated solutions in a single run. The ability of GA to simultaneously search through different regions of a solution space makes it possible to find a diverse set of solutions to difficult problems with non-convex, discontinuous, and multimodal solutions spaces. The crossover operator of GA may exploit structures of good solutions with respect to different objectives to create new non-dominated solutions in unexplored parts of the Pareto front. In addition, most multi-objective GAs do not require the user to prioritize, scale, or weigh objectives. Therefore, GAs have been the most popular heuristic approaches to multi-objective design and optimization problems. Jones et al. [22] reported that $90 \%$ of the approaches to multi-objective optimization aimed to approximate the true Pareto front for the underlying problem. A majority of these used a meta-heuristic technique, and $70 \%$ of all metaheuristics approaches were based on evolutionary approaches. The first multi-objective GA, called Vector Evaluated GA (or VEGA), was proposed by Schaffer [23].

Over the years, numerous techniques have been developed. Some of the well-known and credible algorithms used in many applications and their performances tested in several comparative studies include Vector Evaluated Genetic Algorithm (VEGA), Multi-Objective Genetic Algorithm (MOGA), WeightBased Genetic Algorithm (WBGA), Random Weight Genetic Algorithm (RWGA), Niched Pareto Genetic Algorithm (NPGA), Pareto envelop-based selection algorithm PESA, Pareto Archived Evolution Strategy (PAES), Non-dominated Sorting Genetic Algorithm (NSGA), fast Non-dominated Sorting Genetic Algorithm (NSGA-II), Strength Pareto Evolutionary Algorithm (SPEA), improved Strength Pareto Evolution- 
ary Algorithm (SPEA-2), rankdensity-based genetic algorithm (RDGA), and Dynamic Multi-Objective Evolutionary Algorithm (DMOEA). Generally, multiobjective genetic algorithms differ based on their fitness assignment procedure, elitism, or diversification approaches. Santa Chávez [24] presented a multiobjective ant colony algorithm for the Multi-Depot Vehicle Routing Problem with Backhauls (MDVRPB), where three objectives of traveled distance, traveling times, and total consumption of energy are minimized. An ant colony algorithm is proposed to solve the MDVRPB. The solution scheme allows one to find a set of ordered solutions in Pareto fronts by considering the concept of dominance.

Zhao et al. [25] proposed a multi-objective optimization approach to quality and efficiency in MixedModel Assembly Line (MMAL) by selecting the cycle time at each station. Zhu et al. [26] presented an early attempt to solve one-to-many-to-one Dynamic Pickup-and-Delivery Problem (DPDP) by proposing a multi-objective memetic algorithm called LSH-MOMA, which is a synergy of multi-objective evolutionary algorithm and Locality-Sensitive Hashing-based (LSH) local search. Hajipour et al. [27] presented a Vibration Damping Optimization (VDO) algorithm to solve multi-objective optimization problems for the first time. To do this, fast non-dominated sorting and crowding distance concepts were used in order to find and manage the Pareto-optimal solution.

\subsubsection{NSGA-II: A brief overview}

The notion of Non-dominated Sorting Genetic Algorithm (NSGA) was first suggested by Horn et al. [28]. The main idea behind the non-dominated sorting procedure is that a ranking selection method is used to emphasize the good points, and a niching method is used to maintain a stable subpopulation of the good points. NSGA differs from a simple genetic algorithm only in the way the selection operator works. The crossover and mutation operators remain as usual. The efficiency of NSGA lies in the way that multiple objectives are reduced to a single fitness measure by the creation of number of fronts, sorted according to non-domination. Although NSGA approach has been successfully applied to a number of multi-objective optimization problems, the main criticisms of the NSGA approach have been: (i) its high computational complexity of non-dominated sorting, $O(M N 3)$ where $M$ is the number of objectives and $N$ is the population size; (ii) the lack of elitism; (iii) the need for specifying the tunable sharing parameter. Recently, Deb et al. [21] reported an improved version of NSGA, which they called NSGA-II, to address all of these issues. Specifically, NSGA-II alleviates all the above difficulties by introducing a fast non-dominated sorting procedure with $\mathrm{O}(\mathrm{MN} 2)$ computational complexity, an elitist-preserving approach, and a parameterless niching operator for diversity preservation (crowded comparison operator). NSGA-II also incorporates a simple, yet efficient, penalty parameterless approach to solving constrained multi-objective optimization problems. The constraint-handling approach in NSGA-II uses the binary selection operator, where two solutions are picked up from the population, and the better solution is chosen as follows:

1. If both of the solutions are feasible, choose the solution with a better no-dominated rank;

2. If one is feasible and the other is not, choose the feasible solution;

3. If both of the solutions are infeasible, choose the solution with a smaller overall constraint violation. In the context of constrained multi-objective optimization, Deb modified the definition of domination between two solutions $i$ and $j$ as follows. A solution $i$ is said to constrained-dominate a solution $j$ if any of the following conditions is true.

a) Solution $i$ is feasible and solution $j$ is not.

b) Solutions $i$ and $j$ are both infeasible, but solution $i$ has a smaller overall constraint violation.

c) Solutions $i$ and $j$ are feasible and solution $i$ dominates solution $j$.

The effect of using this constrained-domination principle is that any feasible solution has a better non-domination rank than any infeasible solution. All feasible solutions are ranked according to their nondomination level based on the objective function values. However, between two infeasible solutions, the solution with a smaller constraint violation has a better rank. In fact, when an evolutionary algorithm is applied to multi-objective optimization, two major problems should be considered. The first is how to maintain various populations to prevent premature convergence and achieve a well-distributed trade-off front; the second is how to achieve fitness assignment and selection, separately, to guide the search towards the Pareto-optimal front.

\subsection{Implementation details of the proposed algorithm for multi-objective optimal control problems}

In GA terminology, solution vector $x \in X$ is called an individual or a chromosome. Chromosomes are made of discrete units called genes. Each gene controls one or more features of the chromosome. In the original implementation of GA by Holland, genes are assumed to be binary digits. In later implementations, more varied gene types have been introduced. Normally, a chromosome corresponds to a unique solution $x$ in the solution space. This requires a mapping mechanism between the solution space and chromosomes. 
This mapping is called an encoding. In fact, GA works on the encoding of a problem, not on the problem itself.

In this paper, the chromosomes show the sum of green time duration of the traffic light system. Chromosome length depends on a number of lighting groups inside each intersection.

GA operates with a collection of chromosomes, called a population. The population is normally and randomly initialized. The number of chromosomes inside the population also needs to be decided, because the number of the solutions determines the speed of the optimization and accuracy of the solution found. If too many solutions are generated in the population, then longer duration of time is needed to find the fittest optimization. However, if the number of the solutions is too few or small, the genetic algorithm may face the problem of finding the fittest optimizations.

The key feature of our GA-based optimization strategy is the formulation of regression line, named PDNSGA (Non-Dominated Sorting Genetic Algorithm base on Perpendicular Distance). Linear regression attempts to model the relationship between two variables by fitting a linear equation to observed data. One variable is considered to be an explanatory variable, and the other is considered to be a dependent variable. For example, a modeler might want to relate the weights of individuals to their heights using a linear regression model. The formula for the best-fitting line (or regression line) is $y=m x+b$, where $m$ is the slope of the line, and $b$ is the $y$-intercept. This equation itself is the same one used to find a line in algebra; however, it should be noted that in statistics, the points do not lie perfectly on a line-the line is a model around which the data lie if a strong linear pattern exists.

Linear regression consists of finding the bestfitting straight line through the points. The best-fitting line is called a regression line. The black diagonal line in Figure 3 is the regression line which consists of the predicted score on $Y$ for each possible value of $X$. The vertical lines from the points to the regression line represent the errors of prediction. As we can see, the red point is very close to the regression line; its error of prediction is small. By contrast, the yellow point is much higher than the regression line; therefore, its error of prediction is large.

we may have noticed that we have not specified what is meant by "best-fitting line." By far, the most commonly-used criterion for the best-fitting line is the line that minimizes the sum of the squared errors of prediction. That is the criterion that was used to find the line in Figure 4. The last column in Table 1 shows the squared errors of prediction. The sum of the squared errors of prediction shown in Table 1 is lower than it would be for any other regression line. No-tice that error column sums $\left(Y-Y^{\prime}\right)$ equal zero.

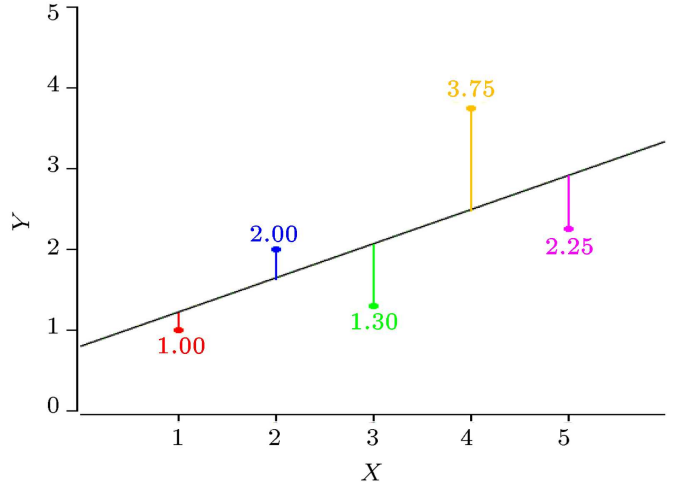

Figure 3. A scatter plot of the example data. The black line consists of the predictions, the points are the actual data, and the vertical lines between the points and the black line represent errors of prediction.

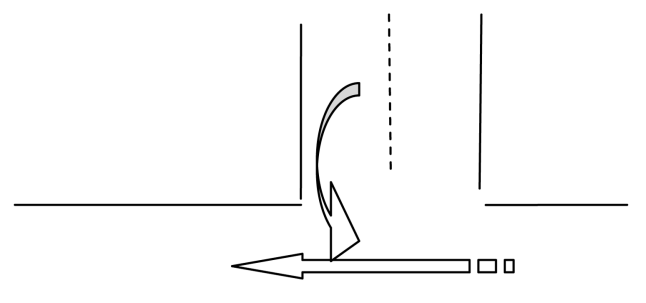

Figure 4. A "two-phase intersection".

Table 1. Example data.

\begin{tabular}{ccccc}
\hline $\boldsymbol{X}$ & $\boldsymbol{Y}$ & $\boldsymbol{Y}^{\prime}$ & $\boldsymbol{Y}-\boldsymbol{Y}^{\prime}$ & $\left(\boldsymbol{Y}-\boldsymbol{Y}^{\prime}\right)^{\mathbf{2}}$ \\
\hline 1 & 1 & 1.21 & -0.21 & 0.044 \\
2 & 2 & 1.635 & 0.365 & 0.133 \\
3 & 1.3 & 2.06 & -0.76 & 0.578 \\
4 & 3.75 & 2.485 & 1.265 & 1.6 \\
5 & 2.25 & 2.91 & -0.66 & 0.436 \\
\hline
\end{tabular}

In this study, we use the specifications of regression line to improve the performance of NSGAII algorithm according to density estimation. To get an estimate of the density of solutions surrounding a particular solution in the population, we calculate the perpendicular distance from the perpendicular line to the regression line passing through the point $(\overline{f 1}, \overline{f 2}, \overline{f 3}, \ldots, \overline{f n})$ where $n$ is the number of objectives. In NSGA-II algorithms, the crowded-comparison operator guides the selection process at various stages of the algorithm toward a uniformly spread-out Pareto optimal front. In this study, we use a new crowdedcomparison operator described below. Although Figure 5 illustrates the crowding-distance computation for two objectives, the procedure is applicable to more than two objectives as well: 


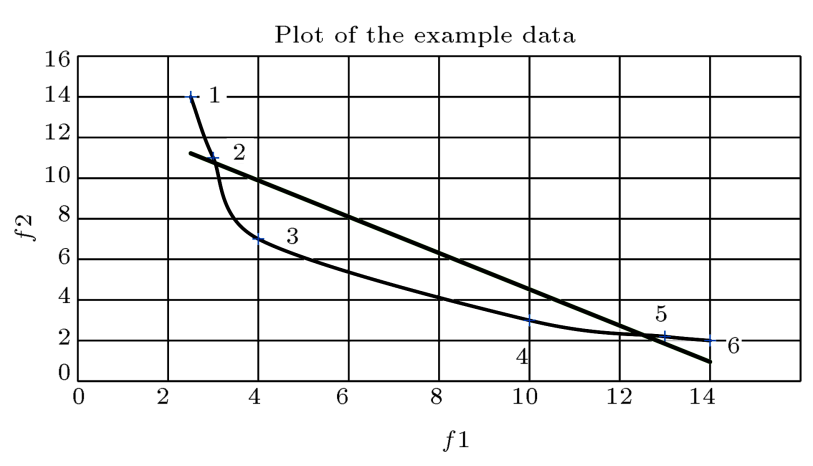

Figure 5. Non-dominated solutions for example data.

Table 2. Mean value.

\begin{tabular}{|c|c|c|c|c|c|c|c|}
\hline \multicolumn{7}{|c|}{ Example data } & \multirow{2}{*}{$\frac{\text { Mean }}{7.8}$} \\
\hline$f 1$ & 14 & 13 & 10 & 4 & 3 & 2.5 & \\
\hline$f 2$ & 2 & 2.2 & 3 & 7 & 11 & 14 & 6.5 \\
\hline
\end{tabular}

- Determine the mean value $(f 1, f 2)$ for each objective in each non-dominated sorting front;

- Calculate the regression line for each non-dominated sorting by the following equation:

$$
\begin{aligned}
& b=\frac{\sum_{i=1}^{N}\left(F 1_{i}-\bar{F} 1\right)\left(F 2_{\mathrm{i}}-\bar{F} 2\right)}{\sum_{i=1}^{N}\left(F 1_{i}-\bar{F} 1\right)}, \\
& a=\bar{F} 2-b \cdot \bar{F} 1, \\
& F 2=b . F 1+a .
\end{aligned}
$$

- Find the equation of a line passing through the mean point $(\overline{f 1}, \overline{f 2})$ and perpendicular to the regression line (red line in Figure 3) using the following equation; two lines are per-pendicular if $b 1 . b 2=-1$. In other words, the slopes of the two lines $\left(b_{1} . b_{2}\right)$ must be negative reciprocals of each other:

$$
y=\frac{-1}{b} x+\left(\overline{f 2}+\frac{1}{b} \overline{f 1}\right) .
$$

- Calculate the distance (blue line in Figure 3) between each solution in the non-dominated sorting $\left(f 1_{i}, f 2_{i}\right)$ and perpendicular to the regression line passing through point $\left(u_{i}, v_{i}\right)$, reached in Eq. (14) using the following equation:

$$
d_{i}=\sqrt{\left(f 1_{i}-\mathrm{U}_{\mathrm{i}}\right)^{2}+\left(f 2_{i}-\mathrm{V}_{\mathrm{i}}\right)^{2}}
$$

For example, we obtained the regression line and perpendicular to the regression line passing through mean point $(\overline{f 1}, \overline{f 2})$ and crowding-distance $\left(d_{i}\right)$ according to Table 2 .
If we use the crowded-comparison operator according to NSGA-II algorithm, we choose point 4 that has lesser crowding distance than point 5 , that is, between two solutions with differing non-domination ranks, we prefer the solution with the lower (better) rank. Otherwise, if both solutions belong to the same front, then we prefer the solution that is located in a lesser crowded region. In the proposed approach, we replace the crowding distance approach with a new approach that eliminates the above difficulties to some extent. The new approach does not require any userdefined parameter for maintaining diversity among population members. The new approach chooses point 5 because it has a greater distance compared to point 4 that is located in a lesser crowded region. Globally, point 5 is better than point 4 as it leads to divergence and extends in non-dominated solutions; it enables decision-makers to evaluate a greater number of alternative solutions. follows:

The details of the algorithm can be outlined as

Step 1. Initially, a random parent population, $P 0$, is created. The population is sorted based on the nondomination.

Each solution is assigned a fitness (or rank) equal to its no-domination level. The usual binary tournament selection, recombination, and mutation operators are used to create an offspring population $Q_{0}$ of size $N$. Let $t=0$;

Step 2. A combined population $R t=P t \cup Q t$ is formed. Population $R t$ is of size $2 N$. Then, population $R t$ is sorted according to nondomination. The best non-dominated set $F i$ is formed;

Step 3. The perpendicular distance operator $\prec$ $n$ is chosen to sort the non-dominated set $F i$ in descending order. The best $N$ members of the set are chosen for the new population $P t+1$;

Step 4. The new population $P t+1$ is now used for selection, crossover, and mutation to create a new population $t+1$.

Step 5. When the termination condition meets, the loop stops; otherwise, $t=t+1$, then turn back to Step 2.

\section{Illustrative example}

The site of our case study chosen for this study is a major arterial in Central Tehran, regularly crossed by cars, trucks, public transportation buses and mopeds. By adopting the formalism introduced in Section 3, the signalized area in Figure 6 is modelled with 8 links, including 4 input and 4 output links.

The model is programmed in the Microsoft Excel 2007 software using the Visual Basic Application 
(VBA). Problem's data given in Table 3 are entered into the application software. We assume the time period studied (AT) to be equal to 30 minute. In other words, in every 30 minutes, road detectors send the traffic data for optimization process. Also, assume that the lower bound of the green time duration per phase, $L_{i}$, is equal to 10 seconds, and the upper bound of the green time duration per phase, $U_{i}$, is equal to 138 seconds.

In this example, there are 268, 435, and 456 solutions. The presented model is solved in order to obtain the optimal solution. The GA parameters are set as follows: $G=100, N=70$, one-point crossover rate $=0.85$, and mutation rate $=0.2$. The program is run on a Pentium 4 PC with CPU $2.8 \mathrm{GHz} ; T G_{1}=10$, $T G_{2}=15, T G_{3}=110, T G_{4}=34$, and its best Pareto solution is obtained according to Figure 2.

\section{Experimental evaluation}

In order to test the convergence of the algorithm, the optimization results (see Figure 7 ) of the proposed algorithm after 50 and 100 generations are compared. As illustrated in Figure 8, the converged speed of the proposed algorithm is relatively fast. After 50 generations, the optimization results have already obtained a relatively good convergence, which is similar to the optimization results after 100 generations. In this way, the evaluation generation can be reduced when it is necessary to consider the calculation time. Although the accuracy of the solution may be lower, it can still be acceptable based on the experimental results.

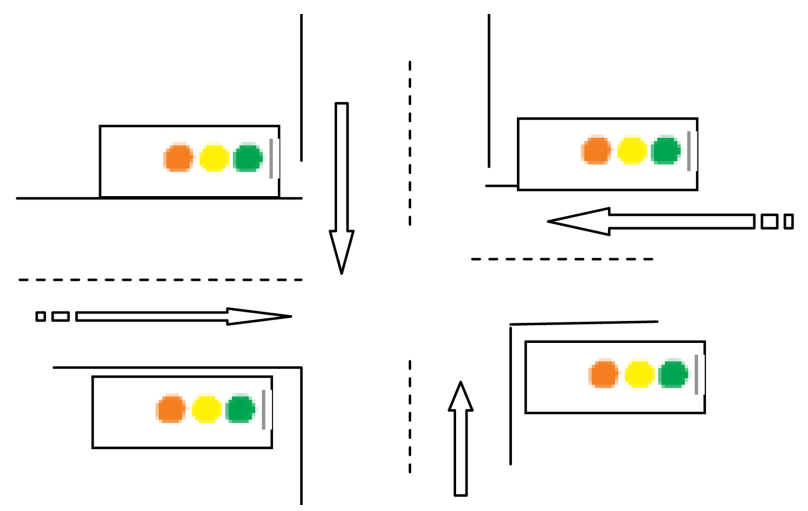

Figure 6. An intersection in traffic network with four phases.
This section evaluates the performances of our proposed GADRL and WBGA [29]. These algorithms are coded and implemented in Excel 2007 by the VBA and are run on a Pentium 4. This section evaluates the performances of the proposed PDNSGA, NSGA-II, MOGA, and WBGA. These algorithms are coded and implemented in Excel 2007 by the VBA and are run on a Pentium $4 \mathrm{PC}$ with $\mathrm{CPU} 2.8 \mathrm{GHz}$ and $512 \mathrm{MB}$ of RAM memory. We use the mean deviation from the ideal point (MDI) as a common performance measure to compare these algorithms computed by:

$$
M D I_{i}=\frac{\sum_{i=1}^{k} \sqrt{\left.\left(F 1_{i}-F 1^{*}\right)_{i}\right)^{2}+\left(F 2_{i}-F 2^{*}\right)^{2}}}{k},
$$

where $F 1^{*}$ and $F 2^{*}$ are the best solutions obtained by each algorithm for a given instance. $F_{1}$ and $F_{2}$ are

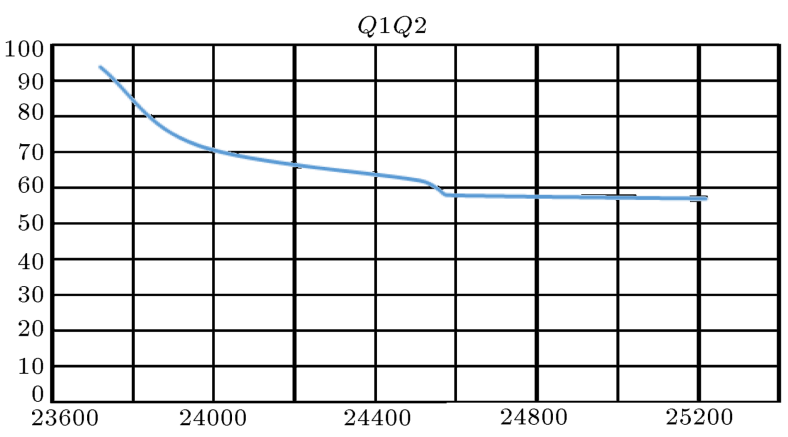

Figure 7. The best Pareto solution of the proposed algorithm.

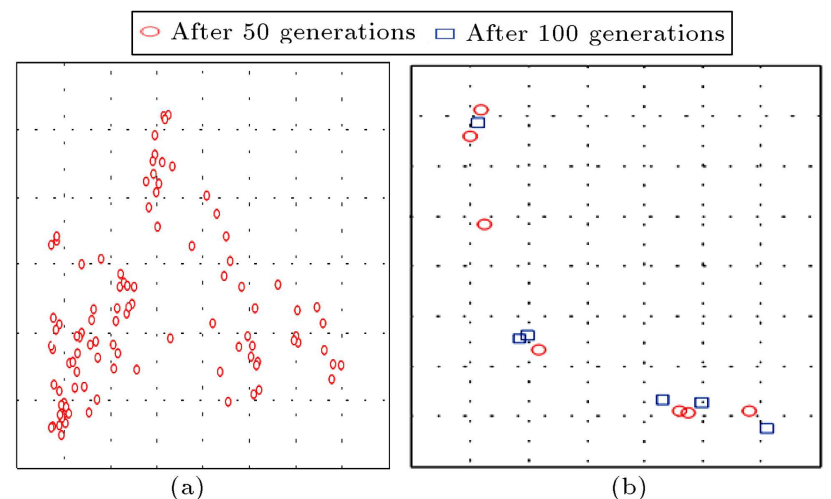

Figure 8. Distribution of optimal solutions after various generations: (a) Initial distribution and (b) distribution of optimal solutions after 50 and 100 generations.

Table 3. The fuzzy entry and exit rate per phase of intersection.

\begin{tabular}{ccccc}
\hline & $\begin{array}{c}\boldsymbol{T} \tilde{\boldsymbol{I}} \boldsymbol{R}_{\boldsymbol{i}} \\
\text { (vehicle/second) }\end{array}$ & $\begin{array}{c}\boldsymbol{T} \tilde{\boldsymbol{I}} \boldsymbol{G}_{\boldsymbol{i}} \\
\text { (vehicle/second) }\end{array}$ & $\begin{array}{c}\boldsymbol{T} \tilde{\boldsymbol{O}} \boldsymbol{G}_{\boldsymbol{i}} \\
\text { (vehicle/second) }\end{array}$ & $\begin{array}{c}\boldsymbol{T} \tilde{\boldsymbol{I}} \boldsymbol{P}_{\boldsymbol{i}} \\
\text { ( man/second) }\end{array}$ \\
\hline Phase 1 & $(5,7,8)$ & $(5,7,8)$ & $(1,2,3)$ & $(5,6,7)$ \\
Phase 2 & $(2,3,4)$ & $(2,3,4)$ & $(3,4,5)$ & $(11,12,13)$ \\
Phase 3 & $(6,7,8)$ & $(6,7,8)$ & $(6,7,9)$ & $(13,14,15)$ \\
Phase 4 & $(3,4,5)$ & $(3,4,5)$ & $(4,5,6)$ & $(8,10,12)$ \\
\hline
\end{tabular}


Individual value plot of $\mathrm{A}, \mathrm{B}, \mathrm{C}, \mathrm{D}$
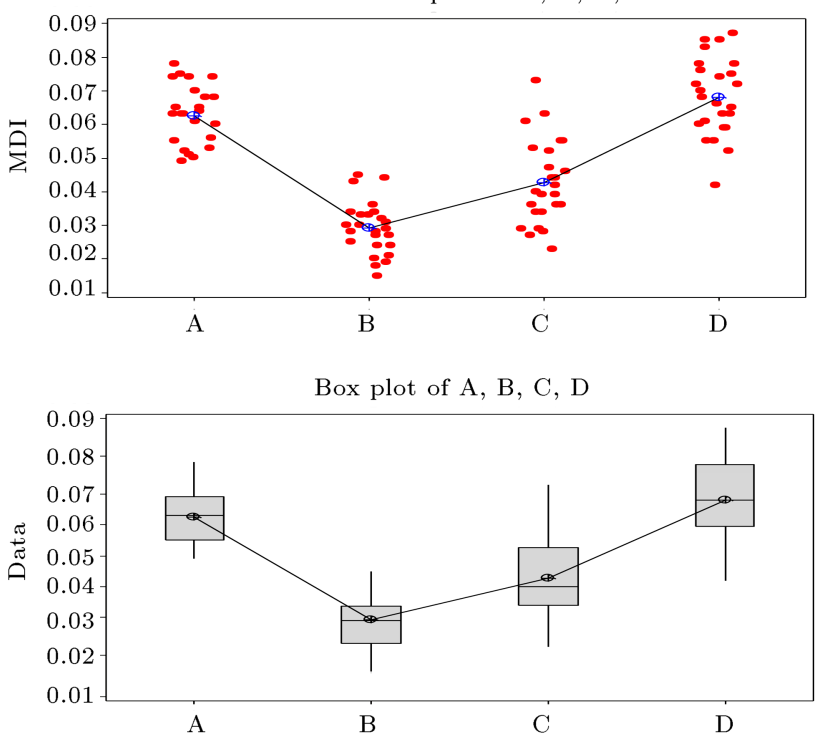

Figure 9. Means plot and LSD intervals for the MOGA (A), PDNSGA (B), NSGA-II (C), and WBGA (D).

the total queue length of vehicles and pedestrians for a given algorithm, respectively, and $k$ is the number of point in each Pareto solution. The PDNSGA, NSGAII, MOGA, and WBGA are implemented with the same parameters for twenty-five times. Their results are analyzed via the analysis of variance (ANOVA) method. The means plot and the Least Significant Different (LSD) interval for the MOGA, PDNSGA, NSGA-II, and WBGA are shown as in Figure 9. It is demonstrated that our proposed algorithm (PDNSGA) gives better outputs than MOGA, NSGA-II, and WBGA do for this problem, statistically.

The proposed PDNSGA procedure has several advantages in speed, flexibility, and accuracy, which would be prominent during its application. Firstly, the proposed PDNSGA procedure runs efficiently. It takes less than half a minute for the proposed PDNSGA procedure to run 50 generations with 5 different random seeds in VBA, while it takes NSGA-II around 3.5 minutes to run 50 generations with only one random seed. Secondly, the proposed GA procedure is flexible, thus can be used in different intersections under different circumstances. The variation includes geometric design of the intersection, vehicle and pedestrian volumes, initial queues, and other traffic relevant parameters. Thus, the signal plan selected by the PDNSGA procedure would be a suitable solution, particularly for that intersection with those traffic characteristics. Moreover, although the analysis period is set as 30 minutes in the tests, it can be set as any positive values, such as 10,5 , or 2 minutes. The shorter the analysis period is, the faster the signal plan responds to the change of traffic demands. With a cycle-length-long analysis period, the PDNSGA procedure can realize a real-time signal plan optimization. However, frequent switches between two pedestrian crossing patterns and big signal timing difference between adjacent cycles should be avoided. Otherwise, either one of them can cause confusion for both drivers and pedestrians, which might bring severe safety issues. In addition, if an object-oriented platform can be set up, the application of the proposed procedure would be even more userfriendly and easier to use. The proposed GA procedure considers pedestrian and vehicle delays in the total user time. However, there are other transportation users as well, e.g. cyclists and buses. Therefore, including the delays of other transportation users into the total user time could be one of the areas for further research.

\section{Conclusion}

A GA optimization procedure was developed in this paper to optimize signal timing of an individual intersection by minimizing total user time, which considers both vehicle and pedestrian delays. In order to verify the proposed optimization procedure, the impact of vehicle and pedestrian volumes on signal plans was tested in a hypothesized intersection. Signal plans selected by the GA procedure are reasonable. Phase lengths increased with the growth of the corresponding critical vehicle or pedestrian volumes.

The main contribution of this paper is presentation of a multi-objective optimization algorithm to optimize traffic signal timing at oversaturated intersection. In the proposed algorithm, maximum throughput and minimum average queue ratio were selected as the optimization objectives to meet the characteristics of oversaturated traffic flow. A problem-solving algorithm, which found an optimal solution, considered the total queue length of vehicles and pedestrians. To solve the given problem, a new genetic algorithm (PDNSGA) was also developed. The model was programmed in the Microsoft Excel 2007 software using the Visual Basic Application (VBA). The proposed algorithm has the capability of searching Pareto front of the multiobjective problem domain. Further jobs should be concerned with the signal timing optimization method for oversaturated coordinated intersections or smallscale road network and real-field applications with the traffic signal controller. The high speed of the proposed algorithm and its quick convergence make it desirable for large scheduling with a large number of phases. Furthermore, we used the mean deviation from the ideal point (MDI) measure to compare the performances of the MOGA, PDNSGA, NSGA-II, and WBGA by the ANOVA method. By considering uncertainty in processing time, this model can be extended to the cases, which can be more realistic.

The proposed GA procedure is only capable of optimizing signal plans for an individual intersection. 
However, there might be several intersections that need to be optimized by minimizing the total user time of both vehicles and pedestrians, especially in a central business district. Therefore, expanding the object of the GA procedure from an individual intersection to a network or arterial could be one of the areas for further research.

\section{References}

1. Ishaque, M.M. and Noland, R.B. "Multimodal microsimulation of vehicle and pedestrian signal timings", In Transportation Research Record 1939, TRB, National Research Council, Washington, D.C, pp. 107$114(2005)$.

2. Ishaque, M.M. and Noland, R.B. "Trade-offs between vehicular and pedestrian traffic using micro-simulation methods", In Transportation Policy, 14, pp. 124-138 (2007).

3. Bhattacharya, P. "Methodology to optimize for pedestrian delay and vehicular delay in a signal network", M.S. Thesis, University of Missouri-Columbia (2004).

4. Bhattacharya, P. and Virkler, M.R. "Optimization for pedestrian and vehicular delay in a signal network", In Transportation Research Record 1939, TRB, National Research Council, Washington, D.C, pp. 115-122 (2005).

5. Li, M., Alhajyassen, W.K.M., and Nakamura, H. "A traffic signal optimization strategy considering both vehicular and pedestrian flows", Presented at the 89th Annual Meeting of the Transportation Research Board, Washington, D.C. (2009).

6. Mehan, S. "Introduction of traffic light controller with fuzzy control system", International Journal of Electronics \& Communication Technology, 2(3), pp. 119-122 (2011).

7. Barzegar, B. "Fuzzy logic controller for traffic signal controller unit system and modelling with colored petri net", Indian Journal of Science and Technology, 4(11), pp. 1420-1428 (2011).

8. Gokulan, B.P. and Srinivasan, D. "Distributed geometric fuzzy multiagent urban traffic signal control", IEEE Trans. Intell. Transp. Syst., 11(3), pp. 714-727 (September 2010).

9. Blum, C. and Roli, A. "Metaheuristics in combinatorial optimization: overview and conceptual comparison", ACM Comput. Surveys (CSUR), 35(3), pp. 268$308(2003)$

10. Holm, P., Tomich, D., Sloboden, J., and Lowrance, C. "Traffic analysis toolbox volume iv: guide-lines for applying corsim microsimulation modeling software", Technical Report, National Technical Information Service-5285 Port Royal Road Springfield, VA 22161, USA-Final Report (2007)

11. Turky, A.M., Ahmad, M.S., Yusoff, M.Z., and Hammad, B.T. "Using genetic algorithm for traffic light control system with a pedestrian crossing", RSKT '09:
Proceedings of the Fourth International Conference on Rough Sets and Knowledge Technology, Berlin, Heidelberg, pp. 512-519 (2009).

12. Odeh, S.M., Mora, A.M., Moreno, M.M., and Merelo, J.J., A Hybrid Fuzzy Genetic Algorithm for an Adaptive Traffic Signal System, Hindawi Publishing Corporation Advances in Fuzzy Sys-tems, Article ID 378156 (2015).

13. Aboudolas, K., Papageorgiou, M., Kouvelas, A., and Kosmatopoulos, E. "A rolling-horizon quadraticprogramming approach to the signal control problem in large-scale congested urban road networks", Transportation Research. Part C: Emerging Technologies, 18, pp. 680-9(2010).

14. Göttlich, S., Herty, M., and Ziegler, U. "Modeling and o timing traffic light settings in road networks", Computers \& Operations Research, 55, pp. 36-51 (2015).

15. Collotta, M., Bellb, L., and Pau, G. "A novel approach for dynamic traffic lights management based on wireless sensor networks and multiple fuzzy logic controllers", Expert Systems with Applications, 42, 13, pp. 5403-5415 (2015).

16. Shahsavari Pour, N., Asadi, H., and Pour Kheradmand, M. "Fuzzy multiobjective traffic light signal optimization", Journal of Applied Mathematics, 7, pp. 01-07 (2013).

17. Duerr, P. "Dynamic right-of-way for transit vehicles: integrated modeling approach for optimizing signal control on mixed traffic arterials. In transportation research broad", Journal of the Transportation Research Board, 1731, pp. 31-39 (2000).

18. Girianna, M. and Benekohal, R.F. "Dynamic signal coordination for networks with oversaturated intersections", In Transportation Research Record 1811, TRB, National Research Council, Washington, D.C., pp. 122-132 (2002).

19. Girianna, M. and Benekohal, R.F. "Using genetic algorithms to design signal coordination for oversaturated networks", In Journal of ITS: Technology, Planning, and Operations, 8(2), pp. 117-129 (2004).

20. Srinivas, N. and Deb, K. "Multiobjective optimization using nondominated sorting in genetic algorithms", $J$ Evol Comput, 2(3), pp. 221-48 (1994).

21. Deb, K., Pratap, A., Agarwal, S., and Meyarivan, T. "A fast and elitist multiobjective genetic algorithm", NSGA-II. IEEE Trans. Evol. Comput,, 6(2), pp. 18297 (2002).

22. Jones, D.F., Mirrazavi, S.K., and Tamiz, M. "Multiobjective metaheuristics an overview of the current state-of-the-art", Eur. J. Oper. Res., 137(1), pp. 1-9 (2002).

23. Schaffer, J.D. "Multiple objective optimization with vector evaluated genetic algorithms", In: Proceedings 
of the International Conference on Genetic Algorithm and Their Applications (1985).

24. Chávez, S., Willmer, J., and Echeverri, E.M.G. "A multi-objective Pareto ant colony algorithm for the multi-depot vehicle routing problem with backhauls", International Journal of Industrial Engineering Computations, 7, pp. 35-48 (2016).

25. Zhao, X., Hsu, Y.C., ChangB, P.C., and Li, L. "A genetic algorithm for the multi-objective optimization of mixed-model assembly line based on the mental workload", Engineering Applications of Artificial Intelligence, 47, pp. 140-146 (2016).

26. Zhu, Z., Xiao, J., He, S., Zhen, J., and Sun, Y. "A multi-objective memetic algorithm based on locality-sensitive hashing for one-to-many-to-one dynamic pickup-and-delivery problem", Information Sciences, 329, pp. 73-89 (2016).

27. Hajipour, V., Mehdizadeh, E., and TavakkoliMoghaddam, R. "A novel Pareto-based multi-objective vibration damping optimization algorithm to solve multi-objective optimization problems", Scientia Iranica E, 21(6), pp. 2368-2378 (2014).

28. Horn, J., Nafpliotis, N., and Goldberg, D.E. "A niched Pareto genetic algorithm for multiobjective optimization", In: Proceedings of the First IEEE Conference on Evolutionary Computation. IEEE World Congress on Computational Intelligence, pp. 27-29 June, 1994. Orlando, FL, USA: IEEE (1994).

29. Spieksma, F.C.R. and Woeginger, G.J. "The no-wait flow-shop paradox", Operation Research Letter, 33, pp. 603-608 (2005).

\section{Biographies}

Hamed Asadi received his BSc in industrial engineering from the Islamic Azad University of Abhar in 2003, then he applied for Science and research branch of Islamic Azad University to study MSc in Industrial Engineering in 2010. He published several research in fuzzy number ranking, flow shop scheduling, traffic signal optimization problem, balance score card, strategic management and multi objective decision making. He received his $\mathrm{PhD}$ degree from Tehran Science and Research University in Iran.

Reza Tavakkoli-Moghaddam received his MS degree in Industrial Engineering from Melbourne University, Australia, and his PhD degree from Swinburne University of Technology, UK. He is currently a Professor of Industrial Engineering at Tehran University, Iran. His research interests include facility layouts and location design, cellular manufacturing systems, sequencing and scheduling, and using meta-heuristics for combinatorial optimization problems. He is the author of over 100 journal papers and 150 papers in conference proceedings.

Nasser Shahsavari Pour is an Assistant Professor of Industrial Engineering at the University of Valie-Asr, Rafsanjan, Iran. He received his MS degree and $\mathrm{PhD}$ degrees from Sharif University of Technology and Tehran Science and Research University in Iran, respectively. He has published more than 40 papers in reputable academic journals (JCR and ISI) and conferences.

Esmail Najafi is an Assistant Professor of Industrial Engineering at the University in Islamic Azad University, Science and Research branch, Iran. He received his $\mathrm{PhD}$ degree from Tehran Science and Research University in Iran, respectively. He has published more than 40 papers in reputable academic journals (JCR and ISI) and conferences. 